\title{
Implementing Implicit Schemes in GENSMAC
}

C.M. OISHI, V.G. FERREIRA, J.A. CUMINATO, A. CASTELO, M.F. TOMÉ, N. MANGIAVACCHI, Departamento de Computação e Estatística, ICMC, USP, 13560-970 São Carlos, SP, Brasil.

\begin{abstract}
Implicit schemes are studied with the purpose of introducing them in the two-dimensional GENSMAC method, for the numerical solution of unsteady newtonian incompressible flows. By using the fractional-step approach, the Freeflow2D simulation system is employed to solve the conservation equations. The viscous terms in the Navier-Stokes equations are implicitly treated via the Implicit Backward (IR), Crank-Nicolson (CN) and Adams-Bashforth/Crank-Nicolson (AB/CN) schemes. The convective terms are explicitly treated by the upwind differencing Variable Order Non-Oscillatory Scheme (VONOS) scheme. For channel and impinging jet flows, the numerical techniques are robust and produce results that compare very well with the analytical solutions.
\end{abstract}

\section{Introduction}

A well-known technique for the numerical simulation of free surface fluid flow problems is the MAC (Marker-And-Cell) method [11]. An improvement on the MAC method was presented by Amsden and Harlow in their paper [1], where they introduced the SMAC (Simplified Marker-And-Cell) method. The main purpose of the SMAC method was to overcome the difficulties of the MAC method in dealing with boundary conditions. In 1994, Tomé and McKee [16] introduced another improvement on the SMAC method known as GENSMAC (GENeralized Simplified Marker-And-Cell) method. The GENSMAC extends the SMAC methodology to cope with arbitrary 2D geometries. Later on, Castelo et al. [6] designed a procedure to introduce surface tension into GENSMAC and, Tomé et al. [17] extended GENSMAC to axisymmetric flows. True to the fact that GENSMAC is an extension of the MAC method, the diffusion term of the Navier-Stokes equations is discretized explicitly. This brings the parabolic stability restriction into play, making the time step very small for some applications, justifying the need for methods with better stability properties.

In 1947, Crank and Nicolson derived, the now famous CN method, an unconditionally stable implicit method to solve the diffusion equation. Approximately, ten years later, Peaceman and Rachford $[8,15]$ introduced the Alternating Direction Implicit method (ADI). Many authors (see, for instance, $[4,12,18]$ ) have contributed to the study and understanding of implicit and semi-implicit methods for solving the conservation equations in fluid flow problems. 
The main purpose of this work is to describe ways of adding implicit schemes to the GENSMAC method for the numerical simulation of transient, viscous, incompressible newtonian flows. For numerically solving the conservation equations, the Freeflow2D [5] simulation environment will be used. This environment is composed of three module: a modeling module (modeler) a simulation module (simulator, which implements the GENSMAC method for solving the Navier-Stokes equations) and the visualization module.

\section{Mathematical Model}

In non-dimensional conservative form, the equations for an incompressible newtonian flow are, respectively, the momentum equations and continuity equation

$$
\begin{gathered}
\frac{\partial \mathbf{u}}{\partial t}+\nabla \cdot(\mathbf{u u})=-\nabla p+\frac{1}{R e} \nabla^{2} \mathbf{u}+\frac{1}{F r^{2}} \mathbf{g}, \\
\nabla \cdot \mathbf{u}=0,
\end{gathered}
$$

where $t$ is time, $\mathbf{u}=[u(x, y, t), v(x, y, t)]$ is the velocity field, $p=p(x, y, t)$ is pressure per unit of mass and $\mathbf{g}=\left(g_{x}, g_{y}\right)$ is the gravity field. The non-dimensional parameters $R e=L U / \nu$ and $F r=U / \sqrt{g L}$ are the Reynolds and Froude numbers, respectively, where $L$ and $U$ are the length and velocity scales and $\nu$ is the kinematic viscosity coefficient of the fluid.

\section{Numerical Method}

The procedure we propose to solve the conservation equations is basically a modification of the GENSMAC method, which uses a fractional step method. Firstly, a provisional velocity field is calculated from the momentum equations and then this velocity field is updated so as to satisfy the continuity equation. When updating the provisional velocity field, a Poisson equation is obtained for an auxiliary potential function $\psi$. In the GENSMAC method, the convective and diffusive terms of the Navier-Stokes equations are discretized explicitly, details of how to proceed can be found in references $[9,16,17]$. Hence, the main modification we shall introduce into GENSMAC is the calculation of the provisional velocity field, which will be made implicit. Three different implicit methods will be tried. The first discretizes the diffusive term using IR, the second uses $\mathrm{CN}$ [2], and the third uses $\mathrm{AB} / \mathrm{CN}[13,14]$. We shall present the provisional velocity field calculation for these three implicit schemes.

As in GENSMAC, from (2.1) we derive the equation

$$
\frac{\partial \tilde{\mathbf{u}}}{\partial t}=-\nabla \cdot(\mathbf{u u})^{n}-\nabla \tilde{p}^{n}+\frac{1}{R e} \nabla^{2} \mathbf{u}^{n}+\frac{1}{F r^{2}} \mathbf{g}^{n},
$$

for the calculation of the provisional velocity field $\tilde{\mathbf{u}}$, where $\tilde{p}$ is a guessed pressure field. For the implicit schemes IR, $\mathrm{CN}$ and $\mathrm{AB} / \mathrm{CN}$, the equation (3.3) takes, 
respectively, one of the following forms:

$$
\begin{gathered}
\frac{\partial \tilde{\mathbf{u}}}{\partial t}-\frac{1}{R e} \nabla^{2} \tilde{\mathbf{u}}=-\nabla \cdot(\mathbf{u u})^{n}-\nabla \tilde{p}^{n}+\frac{1}{F r^{2}} \mathbf{g}^{n} \\
\frac{\partial \tilde{\mathbf{u}}}{\partial t}-\frac{1}{2 R e} \nabla^{2} \tilde{\mathbf{u}}=-\nabla \cdot(\mathbf{u} \mathbf{u})^{n}-\nabla \tilde{p}^{n}+\frac{1}{2 R e} \nabla^{2} \mathbf{u}^{n}+\frac{1}{F r^{2}} \mathbf{g}^{n} \\
\frac{\partial \tilde{\mathbf{u}}}{\partial t}-\frac{1}{2 R e} \nabla^{2} \tilde{\mathbf{u}}=-\frac{3}{2} \nabla \cdot(\mathbf{u u})^{n}+\frac{1}{2} \nabla \cdot(\mathbf{u u})^{n-1}-\nabla \tilde{p}^{n}+\frac{1}{2 R e} \nabla^{2} \mathbf{u}^{n}+\frac{1}{F r^{2}} \mathbf{g}^{n}
\end{gathered}
$$

Hence, one computational cycle consists of updating the discrete variables from the initial time $t_{0}$ to a latter time $t=t_{0}+\delta t$, by using a sequence of inter-related steps, whose details can be found in references $[9,16,17]$.

\section{Discrete Equations}

Equations (2.1) e (2.2) are approximated in a staggered mesh. In this mesh, the pressure is stored at cell centers and the components of the velocity $u$ and $v$ are stored in the middle of the lateral faces (see for instance [9]). As in [16], the diffusion terms and the pressure gradient in equations (3.4)-(3.6) are approximated by central differences, whereas the time derivatives are approximated by forward differences (Euler explicit). Poisson's equation for the potential $\psi$ is approximated by the laplacian five point stencil. The resulting linear system is solved by the conjugate gradient method [10]. The convective terms (denoted here by $C O N V(\cdot)$ ) are discretized by the VONOS scheme [19], which is a bounded upwind technique with limited artificial viscosity. Further details of the implementation of the VONOS scheme can be found in [9]. Summarizing, the conservation equations are approximated by following finite difference equations:

- Components $\tilde{u}$ and $\tilde{v}$ - IR Formulation:

$$
\begin{aligned}
& \tilde{u}_{i+\frac{1}{2}, j}^{n+1}-\frac{\delta t}{R e}\left\{\frac{\tilde{u}_{i+\frac{3}{2}, j}-2 \tilde{u}_{i+\frac{1}{2}, j}+\tilde{u}_{i-\frac{1}{2}, j}}{\delta x^{2}}+\frac{\tilde{u}_{i+\frac{1}{2}, j+1}-2 \tilde{u}_{i+\frac{1}{2}, j}+\tilde{u}_{i+\frac{1}{2}, j-1}}{\delta y^{2}}\right\}^{n+1} \\
& =u_{i+\frac{1}{2}, j}^{n}+\delta t\left\{-\left.\operatorname{CONV}(u)\right|_{i+\frac{1}{2}, j}-\frac{\left(\tilde{p}_{i+1, j}-\tilde{p}_{i, j}\right)}{\delta x}+\frac{1}{F r^{2}} g_{x}\right\}^{n}, \\
& \tilde{v}_{i, j+\frac{1}{2}}^{n+1}-\frac{\delta t}{R e}\left\{\frac{\tilde{v}_{i+1, j+\frac{1}{2}}-2 \tilde{v}_{i, j+\frac{1}{2}}+\tilde{v}_{i-1, j+\frac{1}{2}}}{\delta x^{2}}+\frac{\tilde{v}_{i, j+\frac{3}{2}}-2 \tilde{v}_{i, j+\frac{1}{2}}+\tilde{v}_{i, j-\frac{1}{2}}}{\delta y^{2}}\right\}^{n+1} \\
& =v_{i, j+\frac{1}{2}}^{n}+\delta t\left\{-\left.\operatorname{CONV}(v)\right|_{i, j+\frac{1}{2}}-\frac{\left(\tilde{p}_{i, j+1}-\tilde{p}_{i, j}\right)}{\delta y}+\frac{1}{F r^{2}} g_{y}\right\}^{n} .
\end{aligned}
$$


- Components $\tilde{u}$ and $\tilde{v}$ - CN Formulation:

$$
\begin{aligned}
& \tilde{u}_{i+\frac{1}{2}, j}^{n+1}-\frac{\delta t}{2 R e}\left\{\frac{\tilde{u}_{i+\frac{3}{2}, j}-2 \tilde{u}_{i+\frac{1}{2}, j}+\tilde{u}_{i-\frac{1}{2}, j}}{\delta x^{2}}+\frac{\tilde{u}_{i+\frac{1}{2}, j+1}-2 \tilde{u}_{i+\frac{1}{2}, j}+\tilde{u}_{i+\frac{1}{2}, j-1}}{\delta y^{2}}\right\}^{n+1} \\
& =u_{i+\frac{1}{2}, j}^{n}+\delta t\left\{\frac{1}{2 R e}\left(\frac{u_{i+\frac{3}{2}, j}-2 u_{i+\frac{1}{2}, j}+u_{i-\frac{1}{2}, j}}{\delta x^{2}}+\frac{u_{i+\frac{1}{2}, j+1}-2 u_{i+\frac{1}{2}, j}+u_{i+\frac{1}{2}, j-1}}{\delta y^{2}}\right)\right. \\
& \left.\quad-\left.C O N V(u)\right|_{i+\frac{1}{2}, j}-\frac{\left(\tilde{p}_{i+1, j}-\tilde{p}_{i, j}\right)}{\delta x}+\frac{1}{F r^{2}} g_{x}\right\}^{n}, \\
& \tilde{v}_{i, j+\frac{1}{2}}^{n+1}-\frac{\delta t}{2 R e}\left\{\frac{\tilde{v}_{i+1, j+\frac{1}{2}}-2 \tilde{v}_{i, j+\frac{1}{2}}+\tilde{v}_{i-1, j+\frac{1}{2}}}{\delta x^{2}}+\frac{\tilde{v}_{i, j+\frac{3}{2}}-2 \tilde{v}_{i, j+\frac{1}{2}}+\tilde{v}_{i, j-\frac{1}{2}}}{\delta y^{2}}\right\}^{n+1} \\
& =v_{i, j+\frac{1}{2}}^{n}+\delta t\left\{\frac{1}{2 R e}\left(\frac{v_{i+1, j+\frac{1}{2}}-2 v_{i, j+\frac{1}{2}}+v_{i-1, j+\frac{1}{2}}}{\delta x^{2}}+\frac{v_{i, j+\frac{3}{2}}-2 v_{i, j+\frac{1}{2}}+v_{i, j-\frac{1}{2}}}{\delta y^{2}}\right)\right. \\
& \left.-\left.C O N V(v)\right|_{i, j+\frac{1}{2}}-\frac{\left(\tilde{p}_{i, j+1}-\tilde{p}_{i, j}\right)}{\delta y}+\frac{1}{F r^{2}} g_{y}\right\}^{n} \cdot
\end{aligned}
$$

- Components $\tilde{u}$ e $\tilde{v}$ - AB/CN Formulation:

$$
\begin{aligned}
& \tilde{u}_{i+\frac{1}{2}, j}^{n+1}-\frac{\delta t}{2 R e}\left\{\frac{\tilde{u}_{i+\frac{3}{2}, j}-2 \tilde{u}_{i+\frac{1}{2}, j}+\tilde{u}_{i-\frac{1}{2}, j}}{\delta x^{2}}+\frac{\tilde{u}_{i+\frac{1}{2}, j+1}-2 \tilde{u}_{i+\frac{1}{2}, j}+\tilde{u}_{i+\frac{1}{2}, j-1}}{\delta y^{2}}\right\}^{n+1} \\
& =u_{i+\frac{1}{2}, j}^{n}+\delta t\left\{\frac{1}{2 R e}\left(\frac{u_{i+\frac{3}{2}, j}-2 u_{i+\frac{1}{2}, j}+u_{i-\frac{1}{2}, j}}{\delta x^{2}}+\frac{u_{i+\frac{1}{2}, j+1}-2 u_{i+\frac{1}{2}, j}+u_{i+\frac{1}{2}, j-1}}{\delta y^{2}}\right)\right. \\
& \left.-\left.\frac{3}{2} \operatorname{CONV}(u)\right|_{i+\frac{1}{2}, j}-\frac{\left(\tilde{p}_{i+1, j}-\tilde{p}_{i, j}\right)}{\delta x}+\frac{1}{F r^{2}} g_{x}\right\}^{n}+\delta t\left\{\left.\frac{1}{2} C O N V(u)\right|_{i+\frac{1}{2}, j}\right\}^{n-1}, \\
& \tilde{v}_{i, j+\frac{1}{2}}^{n+1}-\frac{\delta t}{2 R e}\left\{\frac{\tilde{v}_{i+1, j+\frac{1}{2}}-2 \tilde{v}_{i, j+\frac{1}{2}}+\tilde{v}_{i-1, j+\frac{1}{2}}}{\delta x^{2}}+\frac{\tilde{v}_{i, j+\frac{3}{2}}-2 \tilde{v}_{i, j+\frac{1}{2}}+\tilde{v}_{i, j-\frac{1}{2}}}{\delta y^{2}}\right\}^{n+1} \\
& =v_{i, j+\frac{1}{2}}^{n}+\delta t\left\{\frac{1}{2 R e}\left(\frac{v_{i+1, j+\frac{1}{2}}-2 v_{i, j+\frac{1}{2}}+v_{i-1, j+\frac{1}{2}}}{\delta x^{2}}+\frac{v_{i, j+\frac{3}{2}}-2 v_{i, j+\frac{1}{2}}+v_{i, j-\frac{1}{2}}}{\delta y^{2}}\right)\right. \\
& \left.-\left.\frac{3}{2} C O N V(v)\right|_{i, j+\frac{1}{2}}-\frac{\left(\tilde{p}_{i, j+1}-\tilde{p}_{i, j}\right)}{\delta y}+\frac{1}{F r^{2}} g_{y}\right\}^{n}+\delta t\left\{\left.\frac{1}{2} C O N V(v)\right|_{i, j+\frac{1}{2}}\right\}^{n-1} .
\end{aligned}
$$

\section{Numerical Results}

In this section, we present numerical experiments for two problems: channel and impinging jet flows. In all the simulations, we used the final simulation time, specified in the modeling module, as the stopping criterion. 


\subsection{Channel Flow}

In this example, we compare the exact [3] and the approximate solutions for Reynolds numbers of $0.001,0.1$ and 1.0. The approximate solutions are shown at the middle of the channel. The channel model used in the simulations has length $10 \mathrm{~L}$ and width $L$. The mesh used in the numerical simulations has $110 \times 20$ computational cells $\left(\delta_{x}=\delta_{y}=0.10 \mathrm{~m}\right)$. The time simulation for each example was $t=15 \mathrm{~s}$. Two different simulations were performed: For the first, the channel is empty (free surface); for the second, the channel is full of fluid (confined).

The velocity profiles for $u$ obtained by the explicit, IR and CN schemes, together with the analytical profile, are presented in figure 1. Figure 1(a) corresponds to the problem with free surface, simulated with the explicit and IR schemes, and figure 1(b) corresponds to the confined one, simulated by the explicit and CN schemes. It is seen from this figure that the agreement between computed results with these schemes and analytical results can be said to be satisfactory. Moreover, the numerical results by using the explicit and implicit schemes are similar. It should also be observed from this set of figures that this problem was simulated by using the $\mathrm{CN}$ and $\mathrm{AB} / \mathrm{CN}$ schemes for the case with free surface, and IR and $\mathrm{AB} / \mathrm{CN}$ for the confined case. These results are similar to the previous ones (not shown).

It is also worth mentioning that in the case of the free surface flow simulation the minimum time step used was $\delta t=4.0 \times 10^{-3}$ s for the IR scheme (see figure 1 (a)); $\delta t=2.0 \times 10^{-3}$ for the $\mathrm{CN}$ scheme; and $\delta t=2.2 \times 10^{-3} \mathrm{~s}$ for the $\mathrm{AB} / \mathrm{CN}$ scheme, $R e=1$. For the confined problem, the numerical results are also in good agreement with the exact solution. For this problem, the minimum time steps were: $\delta t=1.5 \times 10^{-5}$ for the IR scheme; $\delta t=1.875 \times 10^{-5} \mathrm{~s}$ for the CN scheme (see figure $1(\mathrm{~b})$ ); and $\delta t=1.975 \times 10^{-5} \mathrm{~s}$ for the $\mathrm{AB} / \mathrm{CN}$ scheme, $R e=0.001$.

It is important to note that, when solving the free surface problem, the minimum time step size for the explicit method was $\delta t=1.3 \times 10^{-3} \mathrm{~s}$ for $R e=1$. For the confined problem the values of the minimum time step size is: $\delta t=2.5 \times 10^{-6}$ s for $R e=0.001$.

For a comparison of the CPU time obtained from the explicit and implicit methods, we used the problem of the channel of length $5 \mathrm{~L}$. The mesh used in this case was $100 \times 20$ computational cells $\left(\delta_{x}=\delta_{y}=0.05 \mathrm{~m}\right)$ and $R e=0.1$. Table 1 displays the relative error $\left(E_{r e l}\right)$, the time step and CPU time for this problem. It can be observed from this table that the order of the error for all the methods was the same. The $\delta t$ of the implicit schemes is larger than that the explicit scheme. And the CPU time is considerably larger in the explicit method.

Table 1: Results for confined flow. $R e=0.1$ with simulation time $t=20 \mathrm{~s}$.

\begin{tabular}{|c||c||c||c|}
\hline Method & $E_{\text {rel }}$ & $\delta t(\mathrm{~s})$ & CPU time-(m:s) \\
\hline Explicit & $1.8689 \times 10^{-6}$ & $2.5 \times 10^{-5}$ & $104: 40$ \\
\hline $\mathrm{IR}$ & $1.8689 \times 10^{-6}$ & $2.5 \times 10^{-4}$ & $25: 08$ \\
\hline $\mathrm{CN}$ & $1.8691 \times 10^{-6}$ & $5.0 \times 10^{-4}$ & $22: 41$ \\
\hline $\mathrm{AB} / \mathrm{CN}$ & $1.8691 \times 10^{-6}$ & $5.0 \times 10^{-4}$ & $30: 41$ \\
\hline
\end{tabular}




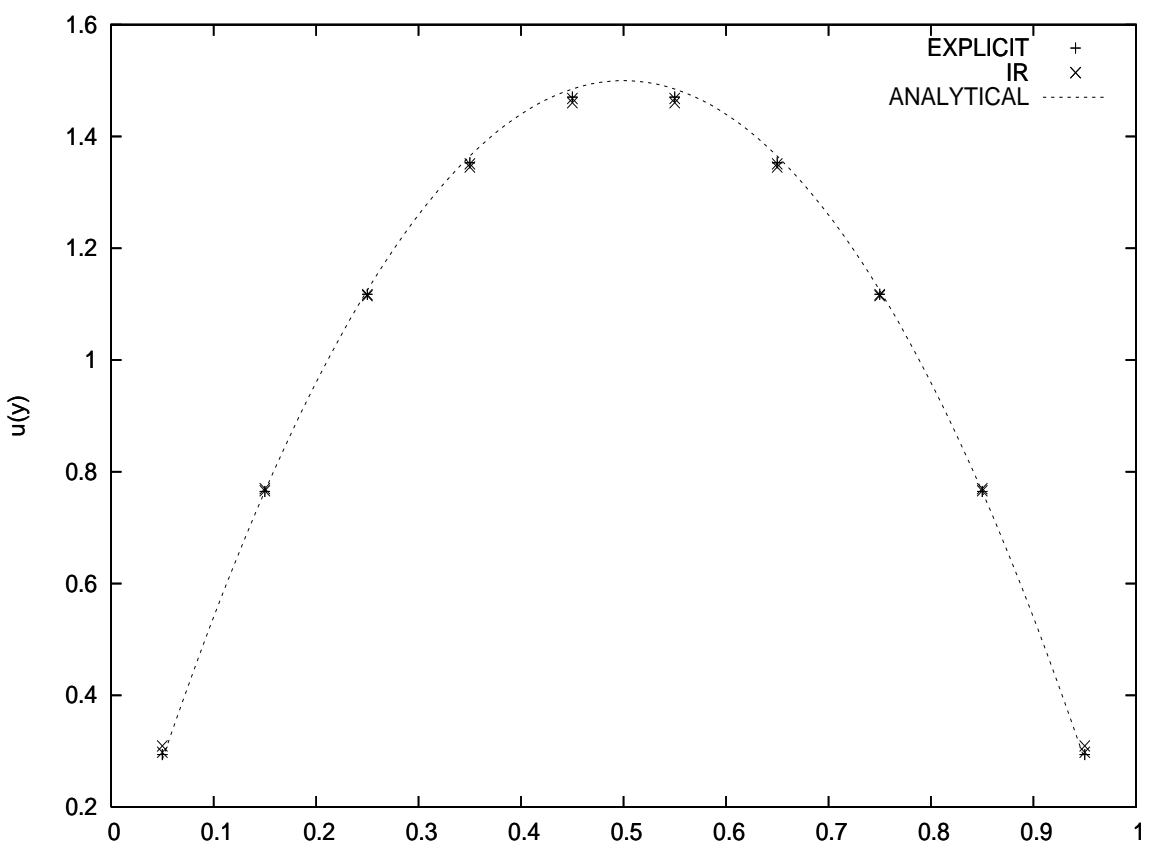

(a)

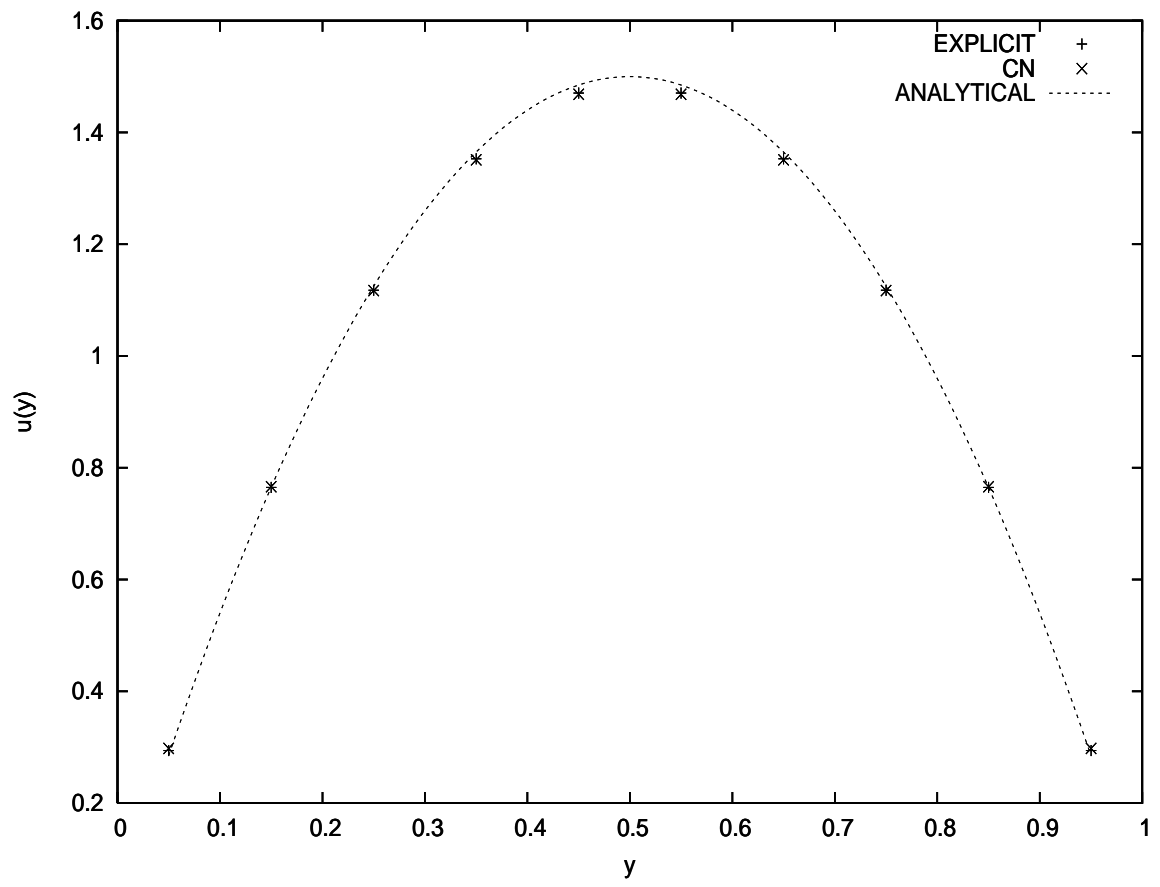

(b)

Figure 1: Comparison between numerical and analytical solutions: (a) IR Scheme with $R e=1$ and (b) CN Scheme with $R e=0.001$, respectively. 
These results show that the procedure allows larger time steps than Euler explicit, as it would be expected. Nevertheless, the presence of the free surface imposes a more stringent restriction on the time step than it would be expected otherwise.

\subsection{Impinging Jet Flow}

The three implicit schemes introduced in previous section were also employed to simulate the flow of an impinging jet onto an impermeable rigid wall, under gravity. For this problem, the Reynolds number, based on the inlet velocity $U=1.0 \mathrm{~m} / \mathrm{s}$ and on the nozzle diameter $L=0.010 \mathrm{~m}$, is $R e=5 \times 10^{3}$ and the Froude number is $F r=3.19254$. The grid used was $800 \times 40$ cells $\left(\delta_{x}=\delta_{y}=0.00050 \mathrm{~m}\right)$. Figure 2 shows a comparison between the numerical solution and the exact solution derived by Watson [20]. This picture shows the non-dimensional free surface of the fluid $(h / 0.5 L)$ plotted against the non-dimensional distance from the origin $(x / 0.5 L) R e^{-1}$. These results were produced by the implicit numerical schemes IR, $\mathrm{CN}$ and $\mathrm{AB} / \mathrm{CN}$ and plotted at time $t=4.0 \mathrm{~s}$. The picture shows good agreement between the numerical solution and Watson's exact solution. It is also worth remarking that this same problem was solved for two other coarser meshes. The results were very similar, indicating grid independence.

\section{Conclusion}

The main purpose of this work is the design and the test of implicit numerical schemes, which can be used in conjunction with the GENSMAC method, for the simulation of transient viscous incompressible newtonian flows. The derived implicit schemes were implemented into the Freeflow2D environment.

Two problems were then simulated, namely: the channel flow and the impinging jet under gravity flow. The numerical results show that the implicit schemes allow for larger time steps when compared with the explicit one, implemented in the original code. For the channel flow problem, the numerical results of the implicit schemes shown very close to the explicit method (in spite of the $\delta t$ for these schemes be larger). This can be seen in Figure 1 or Table 1.

The implicit schemes presented larger overall computational costs due to the need to solve an additional linear system per time step. However, as it was seen in confined problems, the CPU time was much smaller than of the original method.

It should be stressed that the boundary conditions were treated explicitly in this work. Further work will be needed to investigate the influence of the boundary conditions on the stability constraints of implicit schemes. The authors will be

looking into these problems in the near future, including the implicit treatment of the free surface boundary conditions and their influence in the time step bound. 
(a)

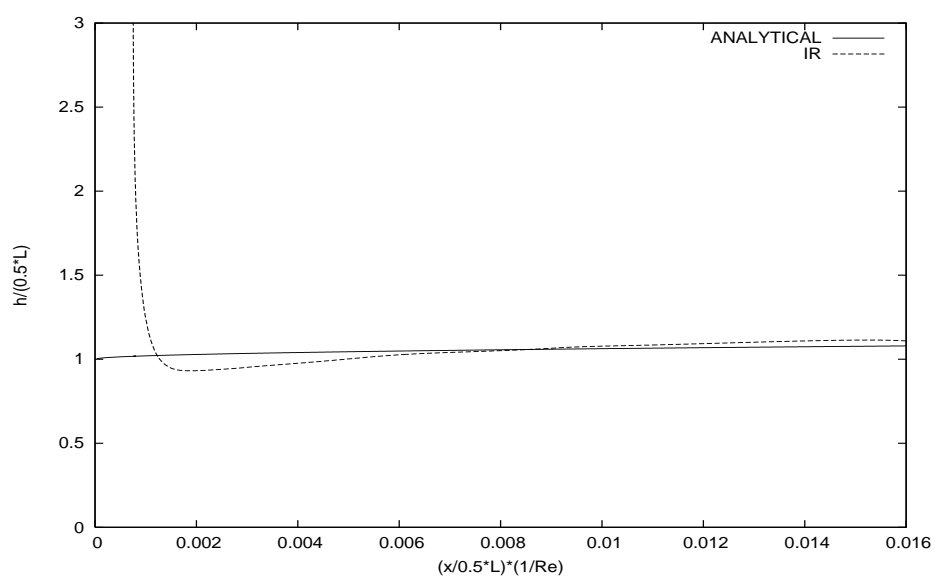

(b)

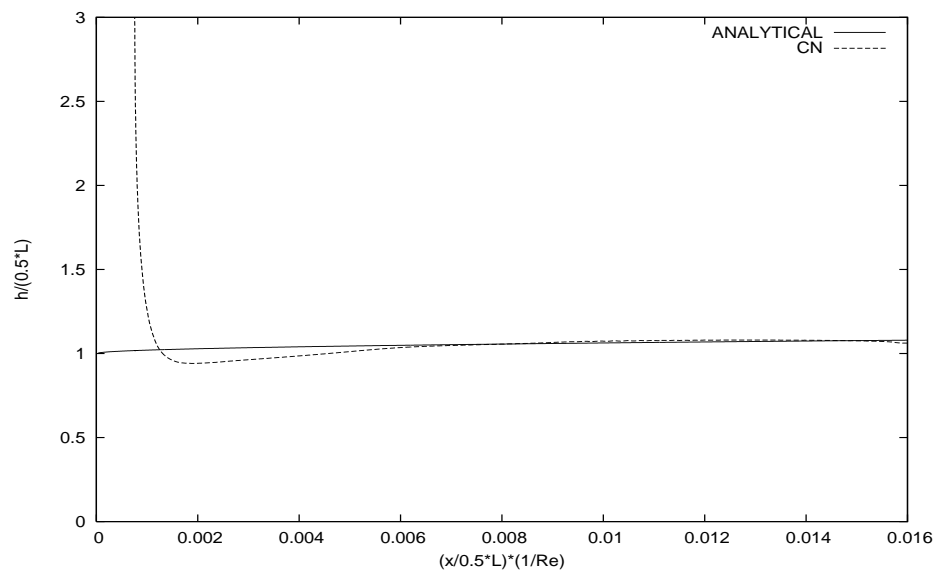

(c)

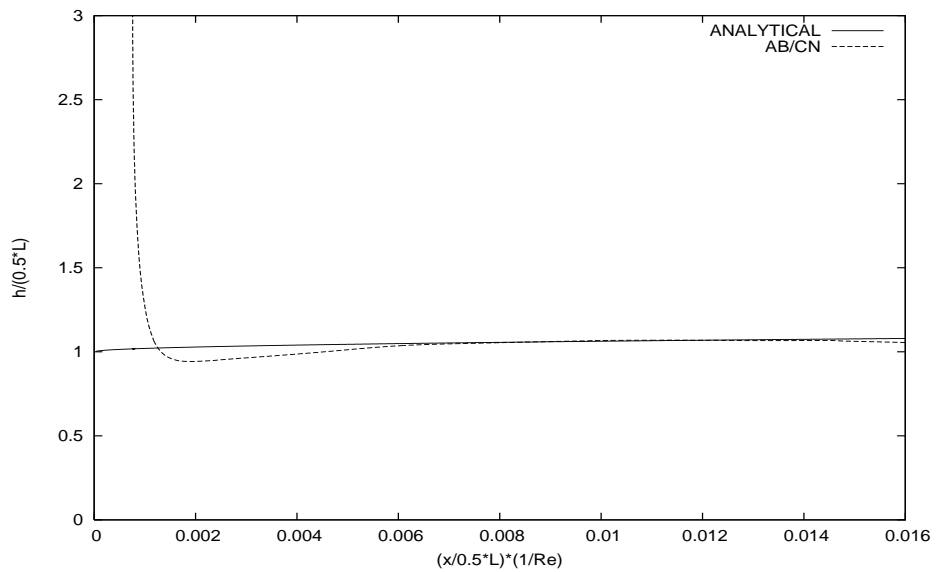

Figure 2: Comparison between Watson's exact solution and the numerical solution for the impinging jet flow, with $R e=5 \times 10^{3}$ : (a) IR Scheme,(b) CN Scheme and (c) $\mathrm{AB} / \mathrm{CN}$ Scheme. 
Resumo. Formulações implícitas são adaptadas na metodologia GENSMAC para problemas bidimensionais, transientes, newtonianos e incompressíveis. Usando um método de passos fracionários, o ambiente de simulação Freeflow2D é utilizado para resolver numericamente as equações de conservação. Os termos difusivos nas equações de Navier-Stokes são tratados implicitamente pelas formulações Implícita Regressiva (IR), Crank-Nicolson (CN) e Adams-Bashforth/Crank-Nicolson (AB/CN). Os termos convectivos são tratados explícitamente pelo esquema upwind de alta ordem e limitado Variable Order Non-Oscillatory Scheme (VONOS). Para escoamentos em canais e jatos sobre superfícies rígidas, as formulações são robustas e fornecem resultados que comparam muito bem com soluções analíticas.

\section{Acknowledgments}

We gratefully acknowledge the support given by FAPESP, contracts 01/12540-2 and $00 / 03385-0$. We are also grateful to the referees for their useful comments to improved the work.

\section{References}

[1] A.A. Amsden and F.H. Harlow, A simplified MAC technique for incompressible fluid flow calculations, J. Comput. Phys., 6 (1970), 332-335.

[2] V. Armenio, An improved MAC method (SIMAC) for unsteady high-Reynolds free surface flows, Int. J. Numer. Meth. Fluid, 24 (1997), 185-214.

[3] G.K. Batchelor, "An Introduction to Fluid Dynamics", Cambridge, 1970.

[4] W.R. Briley and H. McDonald, An overview and generalization of implicit Navier-Stokes algorithms and approximate factorization, Comput. \& Fluid, 30 (2001), 807-828.

[5] A.F. Castelo, M.F. Tomé, J.A. Cuminato, M.L. Cesar and S. McKee, Freeflow: An intregated simulation system for three-dimensional free surface flows, Journal of Comp. and Visual. in Sci., 2 (2000), 199-210.

[6] A.F. Castelo, M.F. Tomé, J.A. Cuminato, J. Oliveira, N. Mangiavacchi, V.G. Ferreira and S. McKee, Surface tension implemention for GENSMAC2D, Revista Brasileira de Ciências Mecânicas, XXIII (2001), 523-532.

[7] H. Chattopadhyay and S.K. Saha, Turbulent flow and heat transfer from a slot jet impinging on a moving plate, (2003), in preparation.

[8] J.J. Douglas and D.W. Peaceman, On the numerical solution of heat conduction problems in two and three space variable, Trans. Am. Math. Soc., 82 (1960), 421-439.

[9] V.G. Ferreira, M.F. Tomé, N. Mangiavacchi, A. Castelo, J.A. Cuminato and S. McKee, High order upwinding and the hydraulic jump, Int. J. Numer. Meth. Fluid, 39 (2002), 549-583. 
[10] W. Hackbush, "Iterative Solution of Large Sparse System of Equations", Springer-Verlag, New York, 1994.

[11] F.H. Harlow and J.E. Welch, Numerical calculation of time-dependent viscous incompressible flow of fluid with free surface, Phys. Fluids, 8 (1965), 2182-2189.

[12] K. Kim, S.J. Baek and H.J. Sung, An implicit velocity decoupling procedure for the incompressible Navier-Stokes equations, Int. J. Numer. Meth. Fluid, 38 (2002), 125-138.

[13] J. Kim and P. Moin, Application of a fractional-step method to incompressible Navier-Stokes equations, J. Comput. Phys., 59 (1985), 308-323.

[14] R. Peyret and T.D. Taylor, "Computional Methods for Fluid Flow", Series in Computional Physics, Springer-Verlag, New York, 1983.

[15] D.W. Peaceman and H.H. Rachford, The numerical solution of parabolic and elliptic diferential equations, J. Soc. Indust. Appl. Math, 3 (1955), 28-41.

[16] M.F. Tomé and S. McKee, GENSMAC: A computational marker-and-cell method for free surface flows in general domains, J. Comput. Phys., 110 (1994), 171-186.

[17] M.F. Tomé, A.F. Castelo, J. Murakami, J.A. Cuminato, R. Minghim, C.F. Oliveira, N. Mangiavacchi and S. McKee, Numerical simulation of axisymmetric free surface flows, J. Comput. Phys., 157 (2000), 441-472.

[18] S. Turek, A comparative study of some time-stepping techniques for the incompressible Naveir-Stokes equations: From fully implicit nonlinear schems to semi-implicit projection methods, (2002), in preparation.

[19] A. Varonos and G. Bergeles, Development and assessment of a variable-order non-oscillatory for convection term discretization, Int. J. Numer. Meths Fluids, 26 (1998), 1-16.

[20] E.J. Watson, The radial spread of a liquid jet over a horinzontal plane, J. Fluid Mechs., 20 (1964), 481-499. 